OPEN ACCESS

Edited by:

Manoj K. Sharma,

Jawaharlal Nehru University, India

Reviewed by:

Shuangxia Jin,

Huazhong Agricultural University,

China

Anindya Bandyopadhyay,

Syngenta, Switzerland

${ }^{*}$ Correspondence:

Anshu Alok

anshualok2@gmail.com

Jitendra Kumar

jitsingh27281@gmail.com

Specialty section

This article was submitted to

Plant Biotechnology,

a section of the journal

Frontiers in Plant Science

Received: 28 August 2019

Accepted: 19 February 2020

Published: 31 March 2020

Citation:

Alok A, Sandhya $D$, Jogam $P$,

Rodrigues $V$, Bhati KK, Sharma $H$ and

Kumar J (2020) The Rise of the

CRISPR/Cpf1 System for Efficient

Genome Editing in Plants.

Front. Plant Sci. 11:264.

doi: 10.3389/fpls.2020.00264

\section{The Rise of the CRISPR/Cpf1 System for Efficient Genome Editing in Plants}

\author{
Anshu Alok ${ }^{1 *}$, Dulam Sandhya ${ }^{2}$, Phanikanth Jogam ${ }^{2}$, Vandasue Rodrigues ${ }^{3}$, \\ Kaushal K. Bhati ${ }^{4}$, Himanshu Sharma ${ }^{5}$ and Jitendra Kumar ${ }^{*}$ \\ ${ }^{1}$ University Institute of Engineering and Technology, Panjab University, Chandigarh, India, ${ }^{2}$ Department of Biotechnology, \\ Kakatiya University, Warangal, India, ${ }^{3}$ Copenhagen Plant Science Centre, Department of Plant and Environmental Sciences, \\ University of Copenhagen, Copenhagen, Denmark, ${ }^{4}$ Louvain Institute of Biomolecular Science, UCLouvain, \\ Louvain-la-Neuve, Belgium, ${ }^{5}$ CSK Himachal Pradesh Agricultural University, Palampur, India, ${ }^{6}$ Department of Plant \\ Pathology, University of Minnesota, Minneapolis, MN, United States
}

Cpf1, an endonuclease of the class 2 CRISPR family, fills the gaps that were previously faced in the world of genome engineering tools, which include the TALEN, ZFN, and CRISPR/Cas9. Other simultaneously discovered nucleases were not able to carry out reengineering at the same region due to the loss of a target site after first-time engineering. Cpf1 acts as a dual nuclease, functioning as an endoribonuclease to process crRNA and endodeoxyribonuclease to cleave target sequences and generate double-stranded breaks. Additionally, Cpf1 allows for multiplexed genome editing, as a single crRNA array transcript can target multiple loci in the genome. The CRISPR/Cpf1 system enables gene deletion, insertion, base editing, and locus tagging in monocot as well as in dicot plants with fewer off-target effects. This tool has been efficiently demonstrated into tobacco, rice, soybean, wheat, etc. This review covers the development and applications of Cpf1 mediated genome editing technology in plants.

Keywords: ZFN, TALEN, Cas9, Cpf1, crRNA, CRISPR, NHEJ, endoribonuclease

\section{INTRODUCTION}

The rapid advancement of genetic engineering tools for functional genomics studies has facilitated a deeper understanding of biological processes. This decade is witnessing revolutionary uses of RNA-guided endonucleases for trait improvement and disease resistance in plants by genome editing (Wang et al., 2014; Ma et al., 2018; Macovei et al., 2018). The Clustered Regularly Interspaced Short Palindromic Repeats (CRISPR) and its associated effector nucleases from bacteria generally belongs to two classes: Class 1 (requires multiple effector proteins) and Class 2 (requires a single effector protein). These two classes have been further diversified into six subtypes. Among them, subtype II contains Cas9 and subtype V contains the Cpf1 effector (Hille and Charpentier, 2016; Koonin et al., 2017). The natural action of the CRISPR/Cas9 system on the viruses has been mimicked and applied as a genetic engineering tool in different kingdoms, including the plant kingdom (DiCarlo et al., 2013; Hou et al., 2013; Hwang et al., 2013; 
Upadhyay et al., 2013; Jiang et al., 2017). Various basic research in the area of CRISPR biology within the last four decades have finally resulted in using CRISPR/Cas9 as a genome-editing tool. This tool has been successfully applied in different crops plants, such as Triticum aestivum (wheat), Oryza sativa (rice), Zea maize (maize), Brassica oleracea, Brassica rapa (mustard), Solanum lycopersicum (tomato), and Solanum tuberosum (potato) as well as fruit plants, such as Musa acuminata (banana), Malus domestica (apple), Citrus X sinensis (orange), and Vitis vinifera L. (grapes) (Jiang et al., 2013; Jia and Wang, 2014; Malnoy et al., 2016; Alok et al., 2017; Shimatani et al., 2017; Kaur et al., 2018; Lee et al., 2019).

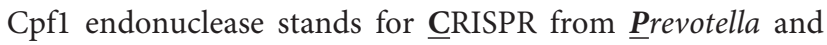
Francisella1, which was previously known as Cas12a. The CRISPR/Cpf1 system has recently gained more popularity as a better substitute for CRISPR/Cas9 and an advanced and more efficient version of a genome-editing tool (Bin Moon et al., 2018). Cpf1 endonuclease is small in size compared to Cas9 and requires shorter CRISPR RNA (crRNA) to work properly (Liu et al., 2017). Cpf1 is an effector nuclease protein guided by a single RNA that binds upstream of the protospacer adjacent motif (PAM) and cuts the DNA at the proximal end of the PAM, far away from the seed region, by introducing 5 base pair (bp) staggered cuts (Zetsche et al., 2015). The CRISPR/Cpf1 system does not require trans-activating crRNA (tracrRNA) while processing Cpf1-associated CRISPR repeats into mature crRNAs (Zetsche et al., 2015; Zhang et al., 2017). This system proficiently cleaves target DNA adjacent to a short T-rich PAM; Cas9, on the other hand, works with a G-rich PAM. Various studies have demonstrated the use of Cpf1 nuclease for targeted genome editing in prokaryotes (Jiang et al., 2017) as well as eukaryotes (Zetsche et al., 2015; Kim H. et al., 2017). Different orthologs of Cpf1 nucleases from various bacteria were isolated and assessed for genome editing, including AsCpf1 and LbCpf1, which were isolated from Acidaminococcus sp. BV3L6 and Lachnospiraceae bacterium ND2006, respectively, and have been extensively used for alterations within a genome (Tóth et al., 2016; Moreno-Mateos et al., 2017; Wang et al., 2017). The main advantage of a CRISPR/Cpf1-mediated genomeediting tool is the reengineering of the desired DNA as the target and that the PAM sequence $\left(5^{\prime}-\mathrm{TTTN}_{-} 3^{\prime}\right)$ remains intact. The PAM sequence may vary according to its origin of ortholog. The Cpf1-database ${ }^{1}$ is an online tool that offers a simple and easy way to find a potential target within the genome and design gRNA, and it can recognize AsCpf1 and LbCpf1 nucleases through DNA recognition sequences (Park and Bae, 2017).

In the present review, we have discussed structural organization and different orthologs of the Cpf1 endonuclease protein and their efficacy toward genome editing in plants. We also discussed sequence variation in crRNA and PAM for different orthologs of Cpf1. Additionally, we have explored different types of CRISPR/Cpf1 vectors used for single and multiple gene editing, transcriptional activation, suppression,

${ }^{1}$ http://www.rgenome.net/cpf1-database/ gene knock-in, and base editing. In addition, we have also assessed the features and limitations of Cpf1.

\section{STRUCTURE OF Cpf1 ENDONUCLEASE}

The Cpf1 endonuclease is a bilobed protein that consists of a helical recognition (REC) lobe; it recognizes a CrRNA-target DNA heteroduplex and a nuclease (NUC) lobe, which cut both strands of DNA. The REC lobe is comprised of two domains; REC1 consists of $13 \alpha$ helices, whereas REC 2 is composed of $10 \alpha$ helices and two $\beta$ strands. The NUC lobe is comprised of RuvC, WED, PI, and Nuc domains. The PI domain interacts with PAM, whereas the Nuc domain cleaves the DNA. The RuvC domain is made up of three motifs: RuvC-I, RuvC-II, and RuvC-III (Yamano et al., 2016).

\section{ORTHOLOGS OF Cpf1}

The lengths of Cpf1 orthologs vary across the bacteria, ranging between $\sim 1,200$ and $\sim 1,500$ amino acids (aa). A sequence analysis of $16 \mathrm{Cpf} 1$ orthologs showed that the $5^{\prime}$ sequence of the direct repeat is much more diverse (Zetsche et al., 2019). The direct repeat sequences of LbCpf1, BpCpf1, and SsCpf1 from Lachnospiraceae bacterium MC2017, Butyrivibrio proteoclasticus, and Smithella sp. SC_K08D17, respectively, were different from the Francisella novicida U112 Cpf1 (FnCpf1) (Zetsche et al., 2015). The Cpf1 from Acidaminococcus spp. BV3L6 (AsCpf1), Lachnospiraceae bacterium ND2006 (LbCpf1), and FnCpf1 have been efficiently used for genome editing in Saccharomyces cerevisiae (Verwaal et al., 2018). The PAM regions of Cpf1 orthologs vary between bacteria to bacteria. For example, the LbCpf1 and AsCpf1 endonucleases require $5^{\prime}-\mathrm{TTTV}-3^{\prime} \mathrm{PAM}$ ( $\mathrm{V}$ is $\mathrm{A}, \mathrm{G}$, or $\mathrm{C}$ ), while FnCpf1 requires $5^{\prime}-\mathrm{TTN}-3^{\prime}$ as PAM site. The size of LbCpf1 is 1,228 aa, which is smaller than AsCpf1 (1,307 aa). In green alga, LbCpf1 has been shown to be more active and efficient in gene editing compared to AsCpf1 (Ferenczi et al., 2017). However, both, LbCpf1 and AsCpf1 are more efficient in their mammalian genome editing than FnCpf1 and MbCpf1 (Tu et al., 2017). Other Cpf1 orthologs isolated from Thiomicrospira sp. Xs5 (TsCpf1), Moraxella bovoculi AAX08_00205 (Mb2Cpf1), Moraxella bovoculi AAX11_00205 (Mb3Cpf1), and Butyrivibrio sp. NC3005 (BsCpf1) were also used to achieve the desired genome editing in human cells (Zetsche et al., 2019). Mb3Cpf1-mediated editing of a target sequence that has $5^{\prime}$-TTTV-3' PAM (where is V may be A, C, or $\mathrm{G}$ ) is comparable to the use of AsCpf1 and LbCpf1 (Zetsche et al., 2019). In rice, AsCpf1 and LbCpf1 were used for genome editing, which showed a higher editing efficiency by LbCpf1 as compared to AsCpf1 (Tang et al., 2017; Li et al., 2018b). Recently, the editing efficiency of LbCpf1 in allotetraploid cotton was tested for the first time. Approximately, 87\% editing efficiency was achieved in T0 cotton plants, which indicates a robust editing efficiency of LbCpf1 in allotetraploid cotton (Li et al., 2019). LbCpf1, also known as Cas12a, was used for genome editing in Nicotiana benthamiana, Solanum lycopersicum, Arabidopsis thaliana, and citrus (Bernabé-Orts et al., 2019; Jia et al., 2019). 


\section{SEQUENCE VARIATION OF crRNA AND PAM FOR Cpf1 ORTHOLOGS}

Almost all variants and orthologs of Cpf1 require a 43nucleotide-long crRNA as compared to Cas9, which requires both tracrRNA and crRNA. The crRNA is made up of a 20 -nucleotide $5^{\prime}$-handle and a 23-nucleotide leader sequence. The leader sequence consists of a seed region and $3^{\prime}$ termini, both of which are complementary to the target region in the genome (Li et al., 2017). Although, the PAM for different orthologs may vary, most of the Cpf1 nucleases require thymine-rich PAM. Earlier, the target range for genome editing using a Cpf1 endonuclease was restricted due to a limitation with PAM recognition sequences. However, researchers have explored different orthologs and engineered variants of $\mathrm{Cpf} 1$ to overcome this limitation, and these are able to identify the alternative PAM. Different studies have demonstrated an increased Cpf1 targeting range using in vitro and in vivo (E. coli) PAM identification assays (Zhang et al., 2017). The two Cpf1 endonucleases, AsCpf1 and LbCpf1, require TTTV as a PAM sequence, where $\mathrm{V}$ can be $\mathrm{A}, \mathrm{C}$, or $\mathrm{G}$ nucleotides. Mutations at position S542R/K607R and S542R/K548V/N552R produced AsCpf1 variants, and these are able to recognize TYCV and TATV PAMs, respectively, where Y can be $\mathrm{C}$ or T (Gao et al., 2017). The AsCpf1 showed increased activity for TTTV PAMs and decreased activity with TTTT PAM (Kim H. K. et al., 2017). The effect of crRNA length also has clear effects on mutation frequency by Cpf1 nucleases. The strong editing activity of Cpf1 requires 17 to $19 \mathrm{bp}$ of guide sequences. However, 4-5 bp at the $3^{\prime}$ end of a 23 bp guide sequence are not necessary for DNA break (Kleinstiver et al., 2016). The shortening of five bp from the $3^{\prime}$ end of a 23 bp-long guide sequence reduced the indel frequency (Kim H. K. et al., 2017; Verwaal et al., 2018). The LbCpf1-RR and LbCpf1-RVR variants relaxed to recognize and work at "CCCC," “TYCV," and "TATG” PAM sites, respectively (Gao et al., 2017). There was a high success rate in rice protoplast for editing CCCC and TYCV PAM sites using an LbCpfl-RR variant and TATG PAM sites using an LbCpf1-RVR variant (Zhong et al., 2018). FnCpf1 variants, i.e., FnCpf1-RR and FnCpf1-RVR, were used in rice for genome editing (Zhong et al., 2018). The FnCpf1 was initially known to edit TTV PAM sites in vitro; however, this did not work in human cells (Zetsche et al., 2015; Tu et al., 2017; Zhong et al., 2018). In plants, only one report showed that FnCpf1 worked for a TTV PAM site (Endo et al., 2016). In rice, FnCpf1 showed activity against a TTV PAM site with VTTV PAM combinations but not against GTTA and GTTC PAM (Zhong et al., 2018).

\section{CRISPR/Cpf1 COMPONENTS AS AN EDITING TOOL}

Genome editing in plants using Cpf1 requires two important components, the Cpf1 protein and a synthetic crRNA. The crRNA fusion with a target DNA with $20 \mathrm{bp}$ results in to a guide RNA. These two components, if they reside on the same vector or two different vectors, can be used for delivery within plant cells (Figure 1). The delivery of the CRISPR/Cpf1 vector into explant is generally carried out using particle bombardment or Agrobacterium mediated. Cpf1 recognizes the base pairing between the guide RNA and target DNA within the genome, and the double-stranded breaks are created by the endonuclease activity.

\section{DeadCpf1 AS A TRANSCRIPTIONAL REPRESSOR/ACTIVATOR}

Cpf1 has a dual nature related to its activity: it acts as RNase on a modified CRISPR array as well as also acting as DNase to break double-stranded DNA (dsDNA) (Wang et al., 2017; Zetsche et al., 2017; Zhang et al., 2017). The Cpf1 endonuclease consists of the RuvC domain, but it lacks the HNH domain, unlike Cas9 endonuclease (Yamano et al., 2016). The mutation within the RvuC domain of Cpf1 leads to loss of nuclease activity for both strands of target DNA (Zhang et al., 2017). The mutation at site E993A within the RuvC domain of AsCpf1 produces a DNAse-dead Cpf1 (ddAsCpf1) (Yamano et al., 2016; Zhang et al., 2017). ddAsCpf1-mediated multiplex gene regulation was demonstrated in Escherichia coli (E. coli) by targeting promoter region. ddAsCpf1 only-without any transcriptional regulator-is enough for targeted repression by its blocking of the transcription initiation and elongation of the desired gene (Zhang et al., 2017). Additionally, ddAsCpf1 can transcriptionally regulate multiple genes by delivering multiple crRNAs, which are subsequently cleaved by its RNase activity. The codon-optimized dead AsCpf1 (D908A) has efficiently been used in bacteria and plants as a transcriptional repressor (Tang et al., 2017; Zhang et al., 2017). In plants, dead Cpf1-mediated transcriptional repressor was successfully used in Arabidopsis and rice. Dead AsCpf1 and LbCpf1 were shown to be efficient for transcriptional repression, and results showed a 10-fold reduction of miR159b transcription (Tang et al., 2017). The transcriptional effector proteins can be fused with catalytically inactive (i.e., dead) Cpf1 and efficiently used in plant and animal systems in a manner similar to dead Cas9. Recently, KRAB, VP64, and VPR domain sequences were fused with dead $\mathrm{AsCpf} 1$ at the $\mathrm{C}$ terminus and were used for targeted repression and activation in human cells (Liu et al., 2017). In another study, a drug-inducible dead LbCpf1, along with transcriptional activators, was used to enhance the expression of multiple genes (Tak et al., 2017).

\section{CRISPR/Cpf1-MEDIATED EDITING IN PLANTS}

CRISPR/Cpf1-mediated genome editing has more potential than other tools such as TALEN, ZnFN, and CRISPR/Cas9. The usefulness of CRISPR/Cpf1 has been successfully demonstrated for targeted mutagenesis in Arabidopsis, rice, tobacco (Nicotiana tabacum), soybean (Glycine max), maize (Zea maize), citrus (Citrus $X$ sinensis), cotton (Gossypium hirsutum), etc. (Endo et al., 2016; Kim H. et al., 2017; Tang et al., 2017; Wang et al., 2017; Xu et al., 2017, 2019; Lee et al., 2019). We have 

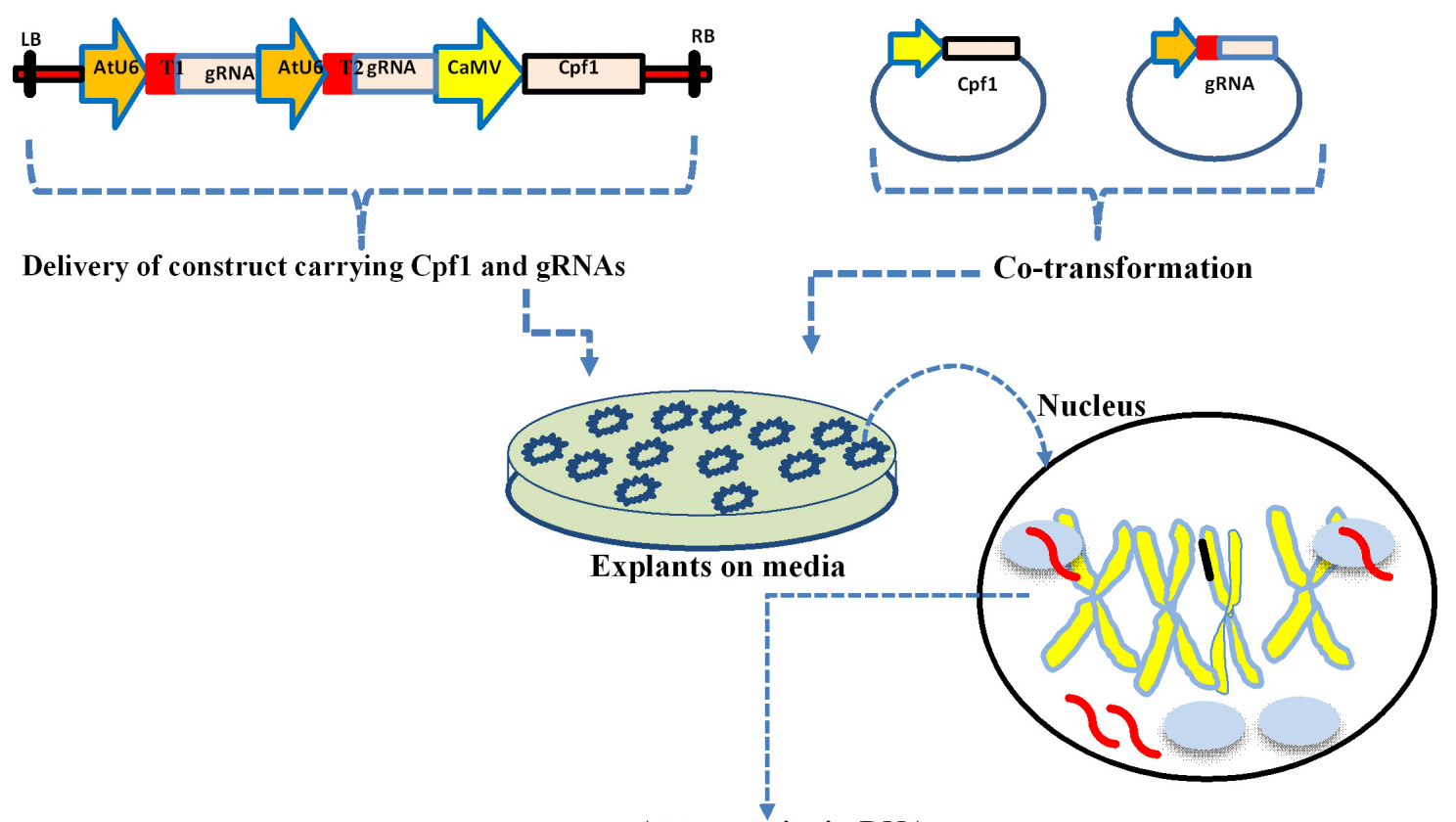

At target site in DNA

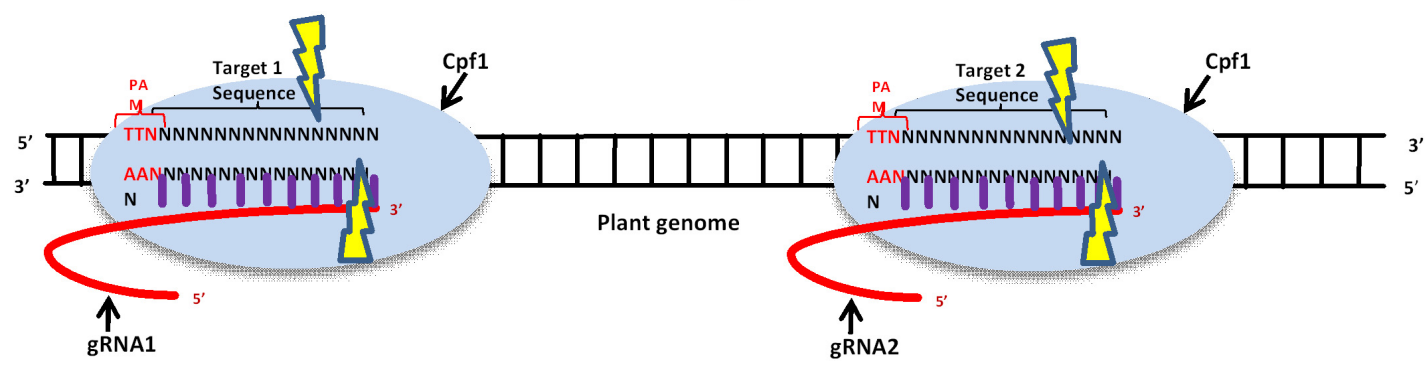

FIGURE 1 | Schematic diagram of delivery of CRISPR/Cpf1 vector into plant cells and the mechanism action of editing. Upper panel depicts single construct and individual vectors carrying Cpf1 and gRNA. LB: left border; T1: Target one; AtU6: Small RNA promoter; CaMV: constitutive promoter, regulates Cpf1; and RB: right border. The delivery of these vectors into plant tissue is usually done by Agrobacterium, gene gun, and PEG mediated co-transformation. The black circle represents the nucleus where Cpf1 and gRNA are expressed. The lower panel depicts the mechanism of editing at a target site within DNA.

tabulated recent works on the Cpf1-mediated gene editing in different plants species in Table 1. In a comparative study of rice, using CRISPR/Cpf1- and CRISPR/Cas9-mediated editing to knock out the Epidermal Patterning Factor like-9 (EPFL9) gene, LbCpf1 showed a higher percentage of mutated T0 plants compared to Cas9. LbCpf1 showed a maximum deletion size of $63 \mathrm{bp}$, whereas Cas9 showed a maximum deletion size of $37 \mathrm{bp}$ (Yin et al., 2017). Endo et al. (2016) showed mutation frequencies of 28.2 and $47.2 \%$ in tobacco and rice, respectively, using CRISPR/Cpf1 at the targeted regions of the genome. Specific and efficient gene insertion or replacement within a genome is also a need in plant genetic engineering. Both LbCpf1 and FnCpf1 endonucleases were used for targeted gene insertion via homology-directed recombination (HDR) in plants. In addition, the targeted insertion frequency for the Cpf1 nuclease was shown to be up to $8 \%$, which is superior compared to other known genome editing endonucleases in rice (Begemann et al., 2017). The size of the gene encoding for Cpf1 is smaller than that of Cas 9 , thus reducing the overall size of the plant transformation vector, which makes for easy packaging and transfer into plant cells.

The vector-carrying construct of the Cpf1 protein along with in vitro synthesized CrRNA can be delivered into plant cells via Agrobacterium, bombardment, and PEG. DNA-free or vector-less editing using the CRISPR/Cpf1 complex has efficiently and frequently been carried out in mammalian cells and can also be performed in plants using PEG-mediated protoplast transformation (Figure 1). In this method, purified Cpf1 proteins, along with in vitro synthesized guide RNA, was transferred into animal cells via PEG or microinjection (Moreno-Mateos et al., 2017). Cpf1 proteins, along with guide RNA complexes, are used as alternative ways for genome editing of plants without introducing DNA into plant cells, and thus referred to as a DNA-free editing system. Recently, Cpf1 proteins and gRNA were efficiently delivered to soybean and wild tobacco protoplasts without T-DNA integration (Kim H. et al., 2017). 
TABLE 1 | List of different application of CRISPR/Cpf1 mediated genome modification in plants.

\begin{tabular}{|c|c|c|c|c|c|}
\hline Plant name & $\begin{array}{l}\text { Transformation } \\
\text { method }\end{array}$ & Binary vector & Selectable marker & Target genes & Reference \\
\hline Rice & Agrobacterium & pCAMBIA & hptII & EPFL9 & Yin et al., 2017 \\
\hline Rice & Agrobacterium & pPZP200 & hptII & $\begin{array}{l}D L \\
\text { ALS, } \\
\text { NCED1 } \\
\text { AO1 }\end{array}$ & Endo et al., 2016 \\
\hline Rice & Agrobacterium & $\mathrm{pHSN} 400$ & hptll & $\begin{array}{l}\text { OsPDS, } \\
\text { OsBEL }\end{array}$ & Xu et al., 2017 \\
\hline Rice (Multiplexing) & Agrobacterium & pCambia & hptll & $\begin{array}{l}\text { OsRLK, } \\
\text { OsBEL }\end{array}$ & Wang et al., 2017 \\
\hline Soybean, Tobacco & $\begin{array}{l}\text { PEG-mediated } \\
\text { protoplasts } \\
\text { transformation }\end{array}$ & p2GW7 & $\begin{array}{l}\text { DAPI and Cy3 } \\
\text { fluorophore probe }\end{array}$ & $\begin{array}{l}F A D 2-1 A \\
F A D 2-1 B\end{array}$ & Kim H. et al., 2017 \\
\hline Tobacco & Agrobacterium & pRI201-AN & nptll & $\begin{array}{l}\text { STF1, } \\
\text { NtPDS }\end{array}$ & Endo et al., 2016 \\
\hline $\begin{array}{l}\text { Allotetraploid } \\
\text { cotton }\end{array}$ & Agrobacterium & pGhRBE3-Cpf1-GhCLA1 & $N A$ & $\begin{array}{l}\text { Cloroplastos alterados } \\
\text { (GhCLA) }\end{array}$ & Li et al., 2019 \\
\hline Maize & Agrobacterium & pYPQ141, 210, 230 & Bialaphos-resistant & Maize glossy2 gene & Lee et al., 2019 \\
\hline Rice & $\begin{array}{l}\text { Protoplasts } \\
\text { transformation }\end{array}$ & STU-Cas12a system & $N A$ & $\begin{array}{l}\text { OsDEP1 } \\
\text { OsROC5 }\end{array}$ & Tang et al., 2019 \\
\hline Rice & Agrobacterium & STU-poly-A vector & $N A$ & $\begin{array}{l}\text { OsPDS } \\
\text { OsDL }\end{array}$ & Xu et al., 2019 \\
\hline $\begin{array}{l}\text { Arabidopsis and } \\
\text { rice }\end{array}$ & $\begin{array}{l}\text { Floral dip and } \\
\text { protoplasts } \\
\text { transformation }\end{array}$ & $\begin{array}{l}\text { dAsCpf1-SRDX and } \\
\text { dLbCpf1-SRDX carrying } \\
\text { vector }\end{array}$ & $N A$ & $\begin{array}{l}\text { OsDEP1 } \\
\text { OsROC5 } \\
\text { OsPDS }\end{array}$ & Tang et al., 2017 \\
\hline Arabidopsis & Floral dip & $\begin{array}{l}\text { enAsCpf1 and ttLbCas } 12 \mathrm{a} \\
\text { carrying vector }\end{array}$ & $N A$ & & Schindele and Puchta, 2019 \\
\hline Rice & $\begin{array}{l}\text { Protoplasts } \\
\text { transformation }\end{array}$ & $\begin{array}{l}\text { Pol II promoter and } \\
\text { ribozyme processing } \\
\text { system }\end{array}$ & $N A$ & DEP1, PDS, and EPFL9 & Zhong et al., 2018 \\
\hline
\end{tabular}

NA, not available.

\section{MULTIPLEX EDITING USING CRISPR/Cpf1}

CRISPR/Cas9-mediated multiplexing has been extensively applied for the alteration of numerous loci in plant genomes. Generally, this multiplexing is performed using two methods. The first one involves expressing many single gRNAs under different small RNA promoters either in same vector or in different vectors. In the second method, multiple single gRNAs are fused with a tRNA recognition sequence, which are expressed as a single transcript under one promoter (Figure 3). Furthermore, these multiple gRNAs are separated into individual gRNAs by endogenous ribonucleases of plant cells (Xie et al., 2015; Tang et al., 2019). In another strategy, the Csy4 gene is expressed with the Cas9 and the Csy4 recognition sequence are fused as a spacer between multiple guide RNAs (Figure 2). The dual activity of Cpfl, cleaving target DNA as well as cleaving its own crRNA, makes it suitable and the easiest way for multiplexing than Cas9 (Zetsche et al., 2015; Fonfara et al., 2016). Unlike Cas9, Cpf1 does not need the support of tracrRNA during maturation of crRNA. Considering these benefits, $\mathrm{Cpf} 1$ has been accepted for the editing of multiple genes using a single crRNA array spaced by mature direct repeats in mammals and plants (Zetsche et al., 2015; Wang et al., 2017).

\section{CRISPR/Cpf1 INCREASED TARGETED AND PRECISE GENE INSERTION}

Gene insertion at a desired and specific position within a plant genome is in demand in plant genetic engineering for the purpose of developing crops with specific traits. This is generally achieved by the well-known plant repair mechanism, i.e., homology-directed repair (HDR). Another repair mechanismnon-homologous end-joining repair (NHEJ)-occurs in plants that generate indel mutations. In the somatic cells of plants, double-stranded breaks are efficiently repaired by NHEJ, which dominates over the HDR (Jiang et al., 2013). Generally, NHEJ results in diverse types of mutations, such as insertion, deletion of few base pairs, chromosome rearrangement, and chromosome relocation. HDR is the key method to carry out precise gene insertion; however, it is limited due to its lower efficiency (Moreno-Mateos et al., 2017).

In past few years, Cas9-mediated gene insertion has been successfully carried out; the insertion rate was 2.5-4.1 and $2.0-2.2 \%$ in maize and rice, respectively. However, the targeted gene insertion in rice using FnCpf1 and LbCpf1 endonucleases was achieved up to 8\% (Begemann et al., 2017; Li et al., 2018a). The indels generated by Cas9 inhibit recurrent cleavage due to mismatches created within the seed region of the target 


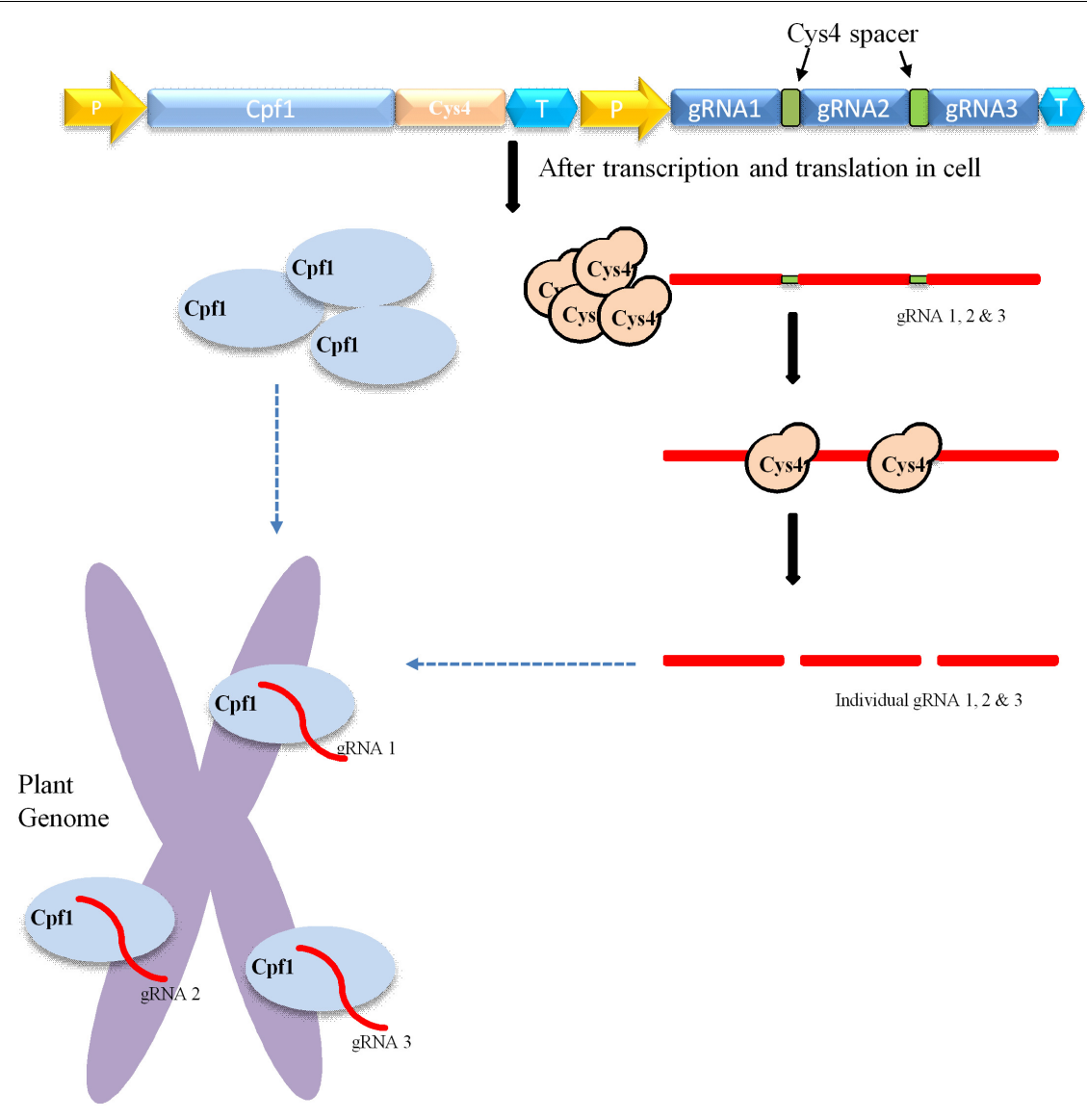

FIGURE 2 | Mechanism of Cpf1-cys4-mediated multiplex genome editing. Construct carrying Cpf1, CRISPR system Yersinia (Csy4), and various gRNAs. The yellow arrow indicates the promoter; the Cpf1 gene is indicated by sky blue; T means the terminator; and green square boxes represent Csy 4 spacers. The delivery and integration of construct into the plant genome leads to the transcription and translation of Cpf1 (sky blue oval) and Csy4 endonuclease (brown circle) within the cell. Csy4 endonuclease acts on spacers (green) and separate individual gRNA1, 2, 3, and so on. Furthermore, these individuals gRNAs bind to target their respective sites within the plant genome, where Cpf1 endonuclease creates double-stranded breaks.

DNA, whereas Cpf1 can repeat cleavages because it cuts $\sim 18$ nucleotides away from the PAM. Due to this nature of Cpf1, it may boost the chance to repair a double-stranded break through HDR (Moreno-Mateos et al., 2017). The rice Chlorophyllidea oxygenase (CAO1) gene, which converts chlorophyll a to chlorophyll $b$, was targeted in rice for gene insertion (Begemann et al., 2017). The disruption of the CAO1 gene leads to yellowing of plants, which was used as a visual marker for gene insertion. The CRISPR/Cpf1 vector and donor template plasmid were cotransformed in rice embryogenic calli using the bombardment method (Begemann et al., 2017).

\section{Cpf1-MediATED BASE EDITING IN GENOME}

DNA base editing requires chimeric protein comprised of an RNA-guided endonuclease and an enzyme able to deaminate an adenine or a cytidine base. The fused deaminating enzyme may be cytidine deaminase (C-to- $\mathrm{T}$ ) or adenine deaminase (A into $\mathrm{G}$ or $\mathrm{A}-\mathrm{T}$ into $\mathrm{G}-\mathrm{C}$ ). APOBEC (apolipoprotein B
mRNA-editing enzyme catalytic polypeptide-like) and AID (activation-induced deaminase) have been used as cytidine deaminase frequently. Initially, base editing was performed in plants with Cas9 that fused a different deaminating domain (Shimatani et al., 2017). However, due to a limitation of the Cas9 PAM site, Cpf1-mediated base editors are in demand and can target T-rich sequences of a plant genome. The first Cpf1-mediated cytidine deaminase base edit was done in human cells in Li et al. (2018c). Li and colleagues generated a Cpf1-mediated base editor by fusing a rat APOBEC1 domain and uracil DNA glycosylase inhibitor to dLbCpf1. They named this base editor dLbCpf1-BE0, and it was effective for sites, T-rich regions, where Cas9 could not bind (Li et al., 2018c).

The dLbCpf1-BE0 showed an editing effect from position eight to $13 \mathrm{bp}$ preceding the PAM (the base next to the PAM was counted as position one). Furthermore, dLbCpf1-BE0 showed low levels of unintended indel mutations and non-C-toT substitutions with a $20-22 \%$ base editing efficiency (Li et al., 2018c). An enhanced Acidaminococcus sp. Cpf1 variant (also known as enAsCas12a) increased the base editing range with 


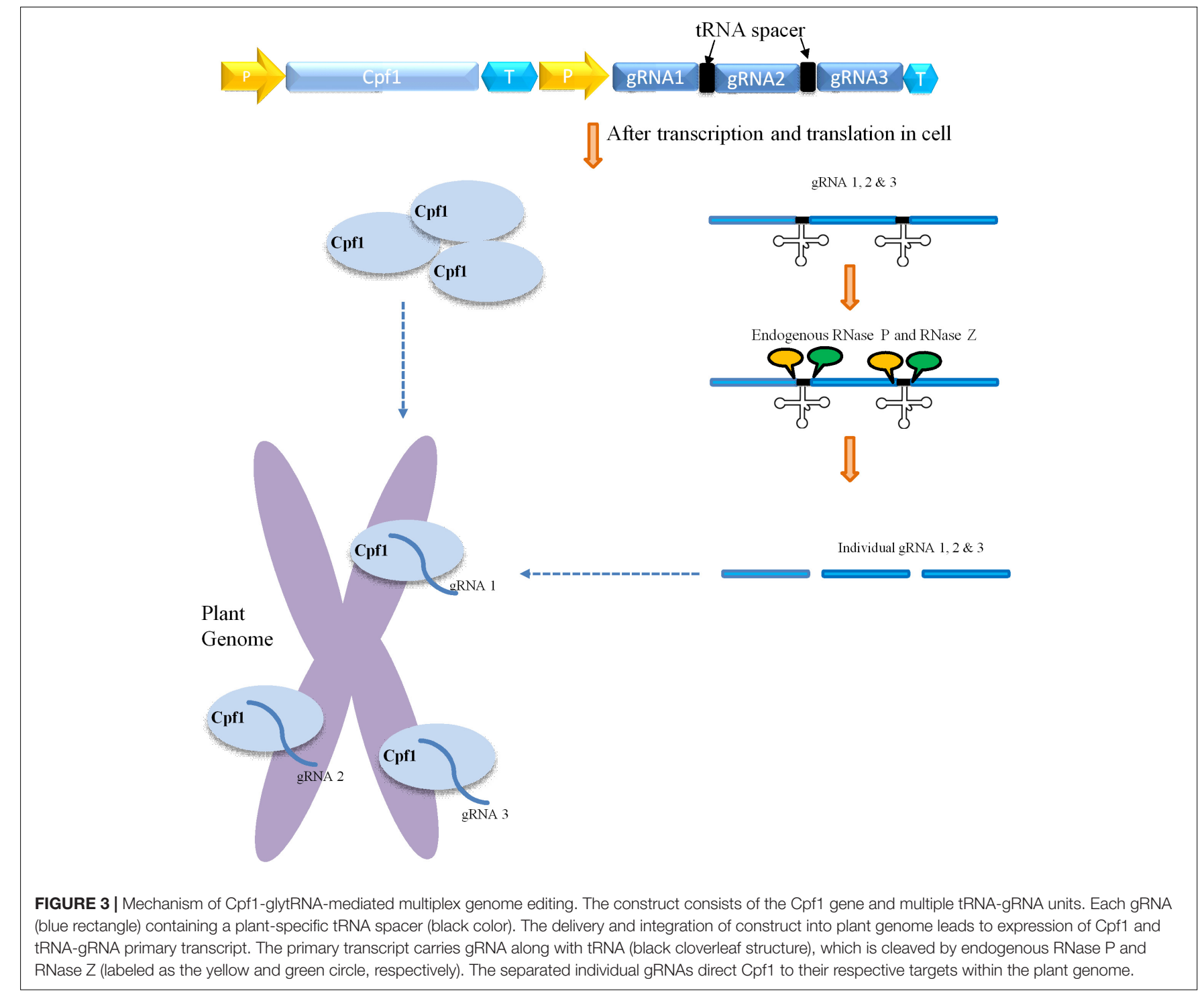

altered PAM in human cells (Kleinstiver et al., 2019). However, to date there has been no report of Cpf1-mediated base editing in plants (Malzahn et al., 2019).

\section{Cpf1 FEATURES AND LIMITATIONS}

Cpf1 endonuclease is small in size and consists of only RuvC-like endonuclease domains in contrast to Cas9 endonuclease, which consists of RuvC-like and HNH domains. CRISPR/Cpf1 cleaves both strands of target DNAs in a staggered pattern, whereas Cas9 cleaves blunt ends. Cas9 requires multiple Pol III promoter to drive various gRNA, whereas Cpf1 needs only one promoter to regulate several crRNA (Zetsche et al., 2017). Cpf1 endonuclease requires only 42-44-nucleotide-long crRNA instead of the engineered 100-nucleotide-long gRNA (tracrRNA and crRNA) desired by Cas9 endonuclease. CRISPR/Cas9 cleaves DNA near PAM,
TABLE 2 | Different features of Cpf1 and Cas9 mediated editing.

\begin{tabular}{|c|c|c|}
\hline Features & CRISPR/Cpf1 & CRISPR/Cas9 \\
\hline Classification & Class 2, Type II system & Class 2, Type V system \\
\hline gRNA & $\begin{array}{l}75 \mathrm{bp} \text { chimeric of } \\
\text { tracrRNA and CrRNA }\end{array}$ & 44 bp CrRNA \\
\hline $\begin{array}{l}\text { PAM recognition } \\
\text { sequence }\end{array}$ & $5^{\prime}-\pi T V-3^{\prime}$ & $5^{\prime}-\mathrm{NGG}-3^{\prime}$ \\
\hline PAM & T-rich PAM site & G-rich PAM site \\
\hline Cleavage & Distal to PAM site & Proximal to PAM site \\
\hline Molecular weight & $157,900 \mathrm{~g} / \mathrm{mol}$ & $163,700 \mathrm{~g} / \mathrm{mol}$ \\
\hline
\end{tabular}

while Cpf1 cleaves DNA distal to PAM without disrupting the 19 bp target sequences. Therefore, Cpf1 gives opportunities for future modifications at the same target site. This suggests that CRISPR/Cpf1-mediated editing may improve the frequency of HDR over NHEJ. Cpf1 endonuclease mediated editing give less errors with controllable insertions due to its sticky ends. 
Various other features make it more suitable for genome modification than Cas9, and these are listed in Table 2.

There are a limitation to Cpf1, and these include shorter crRNA as well as specific temperature requirements during plant genetic transformation (Bernabé-Orts et al., 2019; Lee et al., 2019; Malzahn et al., 2019; Schindele and Puchta, 2019). The crRNAs for Cpf1 are shorter than Cas9, which may lead to the formation of undesirable secondary structures and decreased efficiency, as shown in case of maize (Lee et al., 2019; Malzahn et al., 2019). The maize glossy2 gene was targeted by SpCas9 and LbCpf1 for Agrobacterium-mediated genome editing. Results showed 90\%$100 \%$ editing efficiency in the case of Cas9-edited T0 plants, whereas there was $0 \%-60 \%$ editing efficiency in the case of Cpf1-edited T0 plants.

Cpf1-facilitated genome alteration is temperature sensitive in plants, and this is a major limitation (Lee et al., 2019; Malzahn et al., 2019). The effects of low temperature on the activity of AsCpf1, FnCpf1, and LbCpf1 were documented in Arabidopsis, rice, and maize (Malzahn et al., 2019). For better editing efficiency AsCpf1 requires high temperatures of $28^{\circ} \mathrm{C}$ or above, and LbCpfl showed very low efficiency at $22^{\circ} \mathrm{C}$ that reached to $100 \%$ at $28^{\circ} \mathrm{C}$. A variant of AsCpf 1 , enhanced AsCpf1 or enAsCpf1, showed 2-fold higher activity at lower temperature in human cells (Kleinstiver et al., 2019). A comparison between LbCpf1, Enhanced LbCpf1 (enLbCpf1), and temperature-tolerant LbCpf1 (ttLbCpf1) was conducted at low temperature $\left(22^{\circ} \mathrm{C}\right)$ in Arabidopsis. The result showed

\section{REFERENCES}

Alok, A., Sharma, S., Kumar, J., Verma, S., and Sood, H. (2017). "Engineering in plant genome using agrobacterium: progress and future," in Metabolic Engineering for Bioactive Compounds, eds V. Kalia and A. Saini (Singapore: Springer).

Begemann, M. B., Gray, B. N., January, E., Gordon, G. C., He, Y., Liu, H., et al. (2017). Precise insertion and guided editing of higher plant genomes using Cpf1 CRISPR nucleases. Sci. Rep. 7:11606.

Bernabé-Orts, J. M., Casas-Rodrigo, I., Minguet, E. G., Landolfi, V., GarciaCarpintero, V., Gianoglio, S., et al. (2019). Assessment of Cas12a-mediated gene editing efficiency in plants. Plant Biotechnol. J. 17, 1971-1984. doi: 10.1111/pbi. 13113

Bin Moon, S., Lee, J. M., Kang, J. G., Lee, N. E., Ha, D. I., Kim, D. Y., et al. (2018). Highly efficient genome editing by CRISPR-Cpf1 using CRISPR RNA with a uridinylate-rich 3'-overhang. Nat. Commun. 9:3651.

DiCarlo, J. E., Norville, J. E., Mali, P., Rios, X., Aach, J., and Church, G. M. (2013). Genome engineering in Saccharomyces cerevisiae using CRISPR-cas systems. Nucleic Acids Res. 41, 4336-4343. doi: 10.1093/nar/gkt135

Endo, A., Masafumi, M., Kaya, H., and Toki, S. (2016). Efficient targeted mutagenesis of rice and tobacco genomes using Cpf1 from Francisella novicida. Sci. Rep. 6:38169.

Ferenczi, A., Pyott, D. E., Xipnitou, A., and Molnar, A. (2017). Efficient targeted DNA editing and replacement in Chlamydomonas reinhardtii using Cpf1 ribonucleoproteins and single-stranded DNA. Proc. Natl. Acad. Sci. U.S.A. 114, 13567-13572. doi: 10.1073/pnas.1710597114

Fonfara, I., Richter, H., Bratoviè, M., Le, Rhun, A., and Charpentier, E. (2016). The CRISPR-associated DNA-cleaving enzyme Cpf1 also processes precursor CRISPR RNA. Nature 532, 517-521. doi: 10.1038/nature17945

Gao, L., Cox, D. B., Yan, W. X., Manteiga, J. C., Schneider, M. W., Yamano, T., et al. (2017). Engineered Cpf1 variants with altered PAM specificities. Nat. Biotechnol. 35, 789-792. doi: 10.1038/nbt.3900 that, out of these three, $\mathrm{ttLbCpf1}$ activity was highest at $22^{\circ} \mathrm{C}$ (Schindele and Puchta, 2019).

\section{CONCLUSION}

In this review, we discussed CRISPR/Cpf1 characteristics and its rise to prominence as a powerful genome-editing tool for plants. The Cpf1 orthologous reported from various bacteria and the PAM site variation were significantly useful since they increased the flexibility of the target choice within the genome. Unique features of $\mathrm{Cpf1}$ make it suitable for various applications across diverse plants species, more so than Cas9. Furthermore, CRISPR/Cpf1-mediated knock-in of desired genes showed increased targeted gene insertion. Moreover, the smaller size of Cpf1 may allow for a non-transgenic route, such as viral vector mediated delivery of the CRSIPR/Cpfl, of the genome modification. The potential of the CRISPR/Cpf1 system in trait improvement without a transgene is a well-sought-after approach for the community.

\section{AUTHOR CONTRIBUTIONS}

AA conceptualization the idea. DS, PJ, and AA wrote the manuscript. VR, KB, and JK reviewed and edited. HS drew the image and tabulated the table.

Hille, F., and Charpentier, E. (2016). CRISPR-Cas: biology, mechanisms and relevance. Phil. Trans. R. Soc. B 371:20150496. doi: 10.1098/rstb.2015.0496

Hou, Z., Zhang, Y., Propson, N. E., Howden, S. E., Chu, L. F., Sontheimer, E. J., et al. (2013). Efficient genome engineering in human pluripotent stem cells using Cas9 from Neisseria meningitidis. Proc. Natl. Acad. Sci. U.S.A. 110, 15644-15649. doi: 10.1073/pnas.1313587110

Hwang, W. Y., Fu, Y., Reyon, D., Maeder, M. L., Tsai, S. Q., Sander, J. D., et al. (2013). Efficient genome editing in zebrafish using a CRISPR-Cas system. Nat. Biotechnol. 31, 227-229. doi: 10.1038/nbt.2501

Jia, H., Orboviæ, V., and Wang, N. (2019). CRISPR-LbCas12a-mediated modification of citrus. Plant Biotechnol. J. 17, 1928-1937. doi: 10.1111/pbi. 13109

Jia, H., and Wang, N. (2014). Targeted genome editing of sweet orange using Cas9/sgRNA. PLoS ONE 9:e93806. doi: 10.1371/journal.pone.0093806

Jiang, W., Zhou, H., Bi, H., Fromm, M., Yang, B., and Weeks, D. P. (2013). Demonstration of CRISPR/Cas9/sgRNA-mediated targeted gene modification in Arabidopsis, tobacco, sorghum and rice. Nucleic Acids Res. 41:e188. doi: 10.1093/nar/gkt780

Jiang, Y., Qian, F., Yang, J., Liu, Y., Dong, F., Xu, C., et al. (2017). CRISPRCpf1 assisted genome editing of Corynebacterium glutamicum. Nat. Commun. 8:15179. doi: 10.1038/ncomms15179

Kaur, N., Alok, A., Kaur, N., Pandey, P., Awasthi, P., and Tiwari, S. (2018). CRISPR/Cas9-mediated efficient editing in phytoene desaturase (PDS) demonstrates precise manipulation in banana cv. Rasthali genome. Funct. Integr. Genom. 18, 89-99. doi: 10.1007/s10142-017-0577-5

Kim, H., Kim, S. T., Ryu, J., Kang, B. C., Kim, J. S., and Kim, S. G. (2017). CRISPR/Cpf1-mediated DNA-free plant genome editing. Nat. Commun. 8:14406. doi: 10.1038/ncomms14406

Kim, H. K., Song, M., Lee, J., Menon, A. V., Jung, S., Kang, Y. M., et al. (2017). In vivo high-throughput profiling of CRISPR-Cpf1 activity. Nat. Methods 14, 153-159. doi: 10.1038/nmeth.4104

Kleinstiver, B. P., Sousa, A. A., Walton, R. T., Tak, Y. E., Hsu, J. Y., Clement, K., et al. (2019). Engineered CRISPR-Cas12a variants with increased activities and 
improved targeting ranges for gene, epigenetic and base editing. Nat. Biotechnol. 37, 276-282. doi: 10.1038/s41587-018-0011-0

Kleinstiver, B. P., Tsai, S. Q., Prew, M. S., Nguyen, N. T., Welch, M. M., Lopez, J. M., et al. (2016). Genome-wide specificities of CRISPR-Cas Cpf1 nucleases in human cells. Nat. Biotechnol. 34, 869-874. doi: 10.1038/nbt.3620

Koonin, E. V., Makarova, K. S., and Zhang, F. (2017). Diversity, classification and evolution of CRISPR-Cas systems. Curr. Opin. Microbiol. 37, 67-78. doi: 10.1016/j.mib.2017.05.008

Lee, K., Zhang, Y., Kleinstiver, B. P., Guo, J. A., Aryee, M. J., Miller, J., et al. (2019). Activities and specificities of CRISPR/Cas9 and Cas12a nucleases for targeted mutagenesis in maize. Plant Biotechnol. J. 17, 362-372. doi: 10.1111/pbi.12982

Li, B., Rui, H., Li, Y., Wang, Q., Alariqi, M., Qin, L., et al. (2019). Robust CRISPR/Cpf1 (Cas12a)-mediated genome editing in allotetraploid cotton (Gossypium hirsutum). Plant Biotechnol. J. 17, 1862-1864. doi: 10.1111/pbi. 13147

Li, B., Zhao, W., Luo, X., Zhang, X., Li, C., Zeng, C., et al. (2017). Engineering CRISPR-Cpf1 crRNAs and mRNAs to maximize genome editing efficiency. Nat. Biomed. Eng. 1:0066. doi: 10.1038/s41551-017-0066

Li, S., Li, J., Zhang, J., Du, W., Fu, J., Sutar, S., et al. (2018a). Synthesis-dependent repair of Cpfl-induced double strand DNA breaks enables targeted gene replacement in rice. J. Exp. Bot. 69, 4715-4721. doi: 10.1093/jxb/ery245

Li, S., Zhang, X., Wang, W., Guo, X., Wu, Z., Du, W., et al. (2018b). Expanding the scope of CRISPR/Cpfl-mediated genome editing in rice. Mol. Plant 11, 995-998. doi: 10.1016/j.molp.2018.03.009

Li, X., Wang, Y., Liu, Y., Yang, B., Wang, X., Wei, J., et al. (2018c). Base editing with a Cpf1-cytidine deaminase fusion. Nat. Biotechnol. 36, 324-327. doi: 10.1038/ nbt. 4102

Liu, Y., Han, J., Chen, Z., Wu, H., Dong, H., and Nie, G. (2017). Engineering cell signaling using tunable CRISPR-Cpf1-based transcription factors. Nat. Commun. 8:2095.

Ma, J., Chen, J., Wang, M., Ren, Y., Wang, S., Lei, C., et al. (2018). Disruption of OsSEC3A increases the content of salicylic acid and induces plant defense responses in rice. J. Exp. Bot. 69, 1051-1064. doi: 10.1093/jxb/ erx458

Macovei, A., Sevilla, N. R., Cantos, C., Jonson, G. B., Slamet-Loedin, I., Cermak, T., et al. (2018). Novel alleles of rice eIF4G generated by CRISPR/Cas9-targeted mutagenesis confer resistance to Rice tungro spherical virus. Plant Biotechnol. J. 16, 1918-1927. doi: 10.1111/pbi.12927

Malnoy, M., Viola, R., Jung, M. H., Koo, O. J., Kim, S., Kim, J. S., et al. (2016). DNA-free genetically edited grapevine and apple protoplast using CRISPR/Cas9 ribonucleoproteins. Front. Plant Sci. 7:1904. doi: 10.3389/fpls.2016.01904

Malzahn, A. A., Tang, X., Lee, K., Ren, Q., Sretenovic, S., Zhang, Y., et al. (2019). Application of CRISPR-Cas12a temperature sensitivity for improved genome editing in rice, maize, and Arabidopsis. BMC Biol. 17:9. doi: 10.1186/s12915019-0629-5

Moreno-Mateos, M. A., Fernandez, J. P., Rouet, R., Vejnar, C. E., Lane, M. A., Mis, E., et al. (2017). CRISPR-Cpf1 mediates efficient homology-directed repair and temperature-controlled genome editing. Nat. Commun. 8:2024.

Park, J., and Bae, S. (2017). Cpf1-database: web-based genome-wide guide RNA library design for gene knockout screens using CRISPR-Cpf1. Bioinformatics 34, 1077-1079. doi: 10.1093/bioinformatics/btx695

Schindele, P., and Puchta, H. (2019). Engineering CRISPR/LbCas12a for highly efficient, temperature-tolerant plant gene editing. Plant Biotechnol. J. doi: 10. 1111/pbi.13275 [Epub ahead of print].

Shimatani, Z., Kashojiya, S., Takayama, M., Terada, R., Arazoe, T., Ishii, H., et al. (2017). Targeted base editing in rice and tomato using a CRISPRCas 9 cytidine deaminase fusion. Nat. Biotechnol. 35, 441-443. doi: 10.1038/n bt. 3833

Tak, Y. E., Kleinstiver, B. P., Nuñez, J. K., Hsu, J. Y., Horng, J. E., Gong, J., et al. (2017). Inducible and multiplex gene regulation using CRISPR-Cpf1based transcription factors. Nat. Methods 14, 1163-1166. doi: 10.1038/nmeth. 4483

Tang, X., Lowder, L. G., Zhang, T., Malzahn, A. A., Zheng, X., Voytas, D. F., et al. (2017). A CRISPR-Cpf1 system for efficient genome editing and transcriptional repression in plants. Nat. Plants 3:17018.
Tang, X., Ren, Q., Yang, L., Bao, Y., Zhong, Z., He, Y., (2019). Single transcript unit CRISPR 2.0 systems for robust Cas9 and Cas12a mediated plant genome editing. Plant Biotechnol. J. 17, 1431-1445. doi: 10.1111/pbi.13068

Tóth, E., Weinhardt, N., Bencsura, P., Huszár, K., Kulcsár, P. I., Tálas, A., et al. (2016). Cpf1 nucleases demonstrate robust activity to induce DNA modification by exploiting homology directed repair pathways in mammalian cells. Biol. Direct 11:46. doi: 10.1186/s13062-016-0147-0

Tu, M., Lin, L., Cheng, Y., He, X., Sun, H., Xie, H., et al. (2017). A 'new lease of life': FnCpf1 possesses DNA cleavage activity for genome editing in human cells. Nucleic Acids Res. 45, 11295-11304. doi: 10.1093/nar/gkx783

Upadhyay, S. K., Kumar, J., Alok, A., and Tuli, R. (2013). RNA-guided genome editing for target gene mutations in wheat. G3 3, 2233-2238. doi: 10.1534/g3. 113.008847

Verwaal, R., Buiting-Wiessenhaan, N., Dalhuijsen, S., and Roubos, J. A. (2018). CRISPR/Cpf1 enables fast and simple genome editing of Saccharomyces cerevisiae. Yeast 35, 201-211. doi: 10.1002/yea.3278

Wang, Y., Cheng, X., Shan, Q., Zhang, Y., Liu, J., Gao, C., et al. (2014). Simultaneous editing of three homoeoalleles in hexaploid bread wheat confers heritable resistance to powdery mildew. Nat. Biotechnol 32, 947-951. doi: 10. 1038/nbt.2969

Wang, M., Mao, Y., Lu, Y., Tao, X., and Zhu, J. K. (2017). Multiplex gene editing in rice using the CRISPR-Cpf1 system. Mol. Plant 10, 1011-1013. doi: 10.1016/ j.molp.2017.03.001

Xie, K., Minkenberg, B., and Yang, Y. (2015). Boosting CRISPR/Cas9 multiplex editing capability with the endogenous tRNA-processing system. Proc. Natl. Acad. Sci. U.S.A. 17, 3570-3575. doi: 10.1073/pnas.1420294112

Xu, R., Qin, R., Li, H., Li, D., Li, L., Wei, P., et al. (2017). Generation of targeted mutant rice using a CRISPR-Cpf1 system. Plant Biotechnol. J. 15, 713-717. doi: 10.1111/pbi.12669

Xu, R., Qin, R., Li, H., Li, J., Yang, J. and Wei, P. (2019). Enhanced genome editing in rice using single transcript unit CRISPR-LbCpf1 systems. Plant Biotechnol. J. 17, 553-555. doi: $10.1111 /$ pbi. 13028

Yamano, T., Nishimasu, H., Zetsche, B., Hirano, H., Slaymaker, I. M., Li, Y., et al. (2016). Crystal structure of Cpf1 in complex with guide RNA and target DNA. Cell 165, 949-962. doi: 10.1016/j.cell.2016.04.003

Yin, X., Biswal, A. K., Dionora, J., Perdigon, K. M., Balahadia, C. P., Mazumdar, S., et al. (2017). CRISPR-Cas9 and CRISPR-Cpf1 mediated targeting of a stomatal developmental gene EPFL9 in rice. Plant Cell Rep. 36, 745-757. doi: 10.1007/ s00299-017-2118-z

Zetsche, B., Gootenberg, J. S., Abudayyeh, O. O., Slaymaker, I. M., Makarova, K. S., Essletzbichler, P., et al. (2015). Cpf1 is a single RNA-guided endonuclease of a class 2 CRISPR-Cas system. Cell 163, 759-771. doi: 10.1016/j.cell.2015.09.038

Zetsche, B., Heidenreich, M., Mohanraju, P., Fedorova, I., Kneppers, J., DeGennaro, E. M., et al. (2017). Multiplex gene editing by CRISPR-Cpf1 using a single crRNA array. Nat. Biotechnol. 35:178. doi: 10.1038/nbt0217-178b

Zetsche, B., Strecker, J., Abudayyeh, O. O., Gootenberg, J. S., Scott, D. A., and Zhang, F. (2019). A survey of genome editing activity for 16 Cpf1 orthologs. Keio J. Med. doi: 10.2302/kjm.2019-0009-OA

Zhang, X., Wang, J., Cheng, Q., Zheng, X., Zhao, G., and Wang, J. (2017). Multiplex gene regulation by CRISPR-ddCpf1. Cell Discov. 3:17018.

Zhong, Z., Zhang, Y., You, Q., Tang, X., Ren, Q., Liu, S., et al. (2018). Plant genome editing using FnCpf1 and LbCpf1 nucleases at redefined and altered PAM sites. Mol. Plant 11, 999-1002. doi: 10.1016/j.molp.2018.03.008

Conflict of Interest: The authors declare that the research was conducted in the absence of any commercial or financial relationships that could be construed as a potential conflict of interest.

Copyright (c) 2020 Alok, Sandhya, Jogam, Rodrigues, Bhati, Sharma and Kumar. This is an open-access article distributed under the terms of the Creative Commons Attribution License (CC BY). The use, distribution or reproduction in other forums is permitted, provided the original author(s) and the copyright owner(s) are credited and that the original publication in this journal is cited, in accordance with accepted academic practice. No use, distribution or reproduction is permitted which does not comply with these terms. 